\title{
La respuesta educativa del PT en un contexto de inclusión para el alumnado sordo y oyente
}

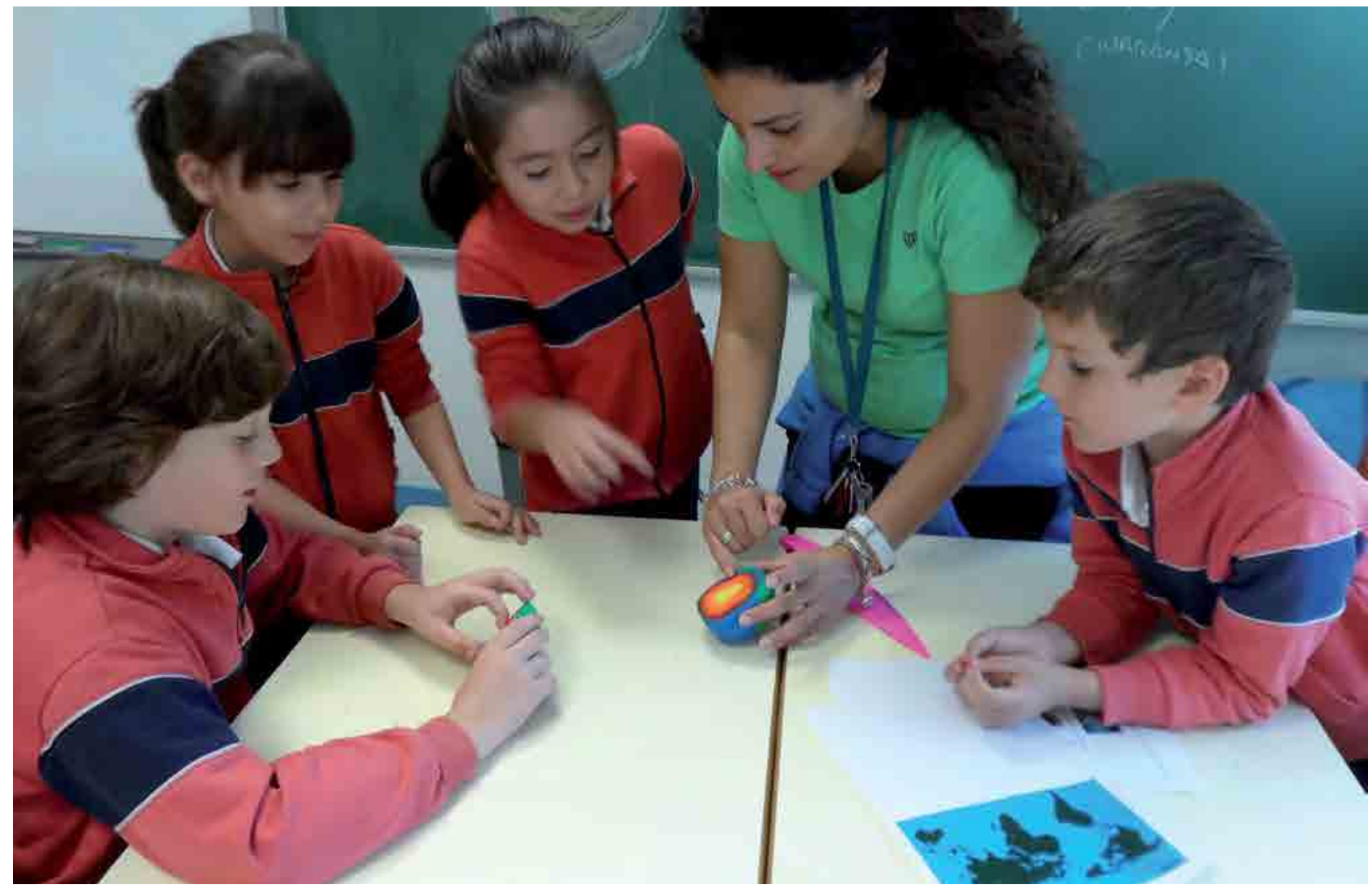

En el artículo presentamos la experiencia educativa que llevamos a cabo en el centro educativo Ponce de León, donde apostamos por una metodología inclusiva en la que conviven personas sordas y oyentes. Para atender a esta diversidad contamos con dos tutores por aula que realizan las labores de tutor y PT. De manera que el apoyo educativo no siempre se realiza fuera del aula sino que en muchas ocasiones ocurre dentro de ella.

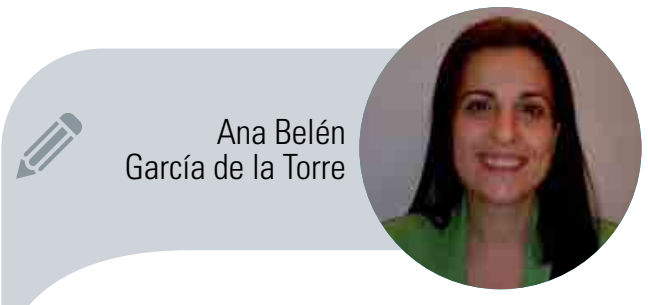

Centro Educativo Ponce de León. Fundación Monte Madrid bgarciat@ponceleon.org

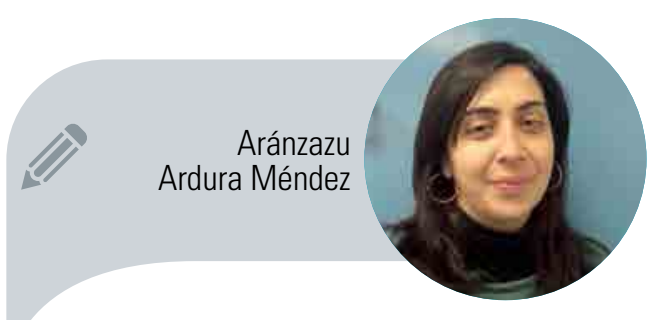

Centro Educativo Ponce de León. Fundación Monte Madrid aardurap@ponceleon.org 


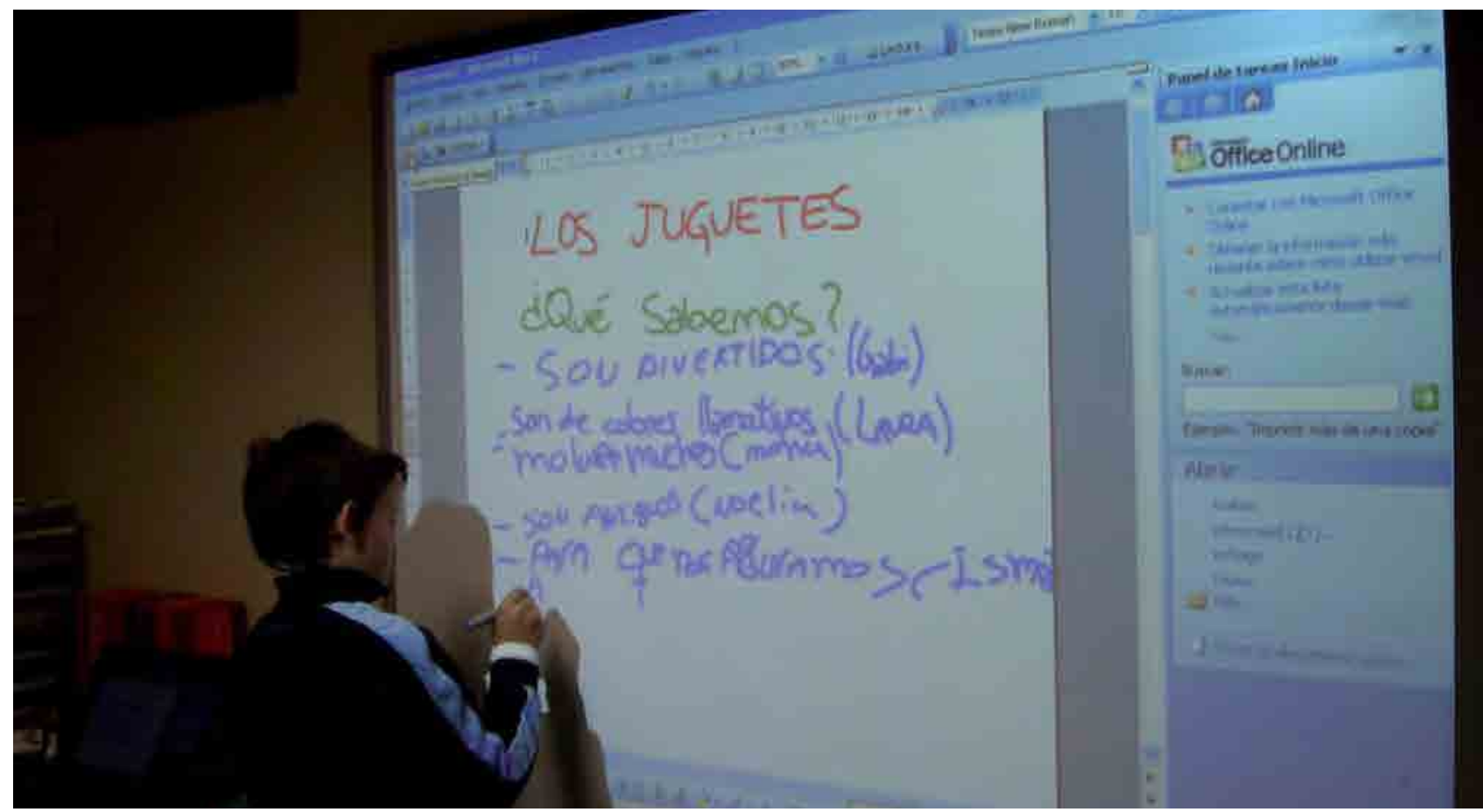

Elaboramos conjuntamente el índice del proyecto de investigación sobre el que vamos a aprender.

\section{Nuestro contexto educativo}

Desde el año 2004, con el asesoramiento del equipo específico de discapacidad auditiva de la Comunidad de Madrid, el centro puso en marcha una experiencia educativa singular en las etapas de Educación Infantil (segundo ciclo, 3-6 años) y Educación Primaria, basada en los modelos bilingües de enseñanza a niños sordos (lengua de signos española-lengua castellana).

Este proyecto está basado en un modelo escolar inclusivo, entendiendo por escuela inclusiva aquélla que persigue cualificar la propia escuela para dar respuesta a todo el alumnado, sordo y oyente, y fijamos nuestras prioridades en la manera de presentar y abordar el currículo, en la metodología que utilizamos y en la organización, para que todos tengan acceso a aprendizajes, sean cuales sean sus necesidades.

Nuestra meta es dar una respuesta educativa de calidad al alumnado oyente y sordo para lo que es imprescindible trabajar conjuntamente en la misma aula, compartiendo espacios, actividades, formando personas respetuosas, tolerantes y solidarias con la diferencia.

Para la consecución de estos objetivos, el proyecto educativo de nuestro centro tiene dos pilares básicos: la educación bilingüe (lengua castellana-lengua de signos española) y la educación compartida (alumnos sordos y oyentes).
Hablar de bilingüismo referido al mundo de las personas sordas, no sólo es un hecho cotidiano deseable, sino que es visto como una necesidad. En todos los países hay individuos bilingües y comunidades bilingües, y en todas ellas hay personas sordas que usan la lengua de signos y que aprenden la lengua de su país, porque consideran que han de ser competentes en lengua oral y escrita para su plena integración social.

En el caso de los alumnos sordos no es tanto una elección personal como una necesidad imperiosa para su verdadera integración en la sociedad a la que pertenecen, integración que debe conseguirse sin detrimento de la lengua que les puede permitir desarrollarse y aprender de forma más natural y construirse una identidad: la lengua de signos.

Parece evidente, entonces, la necesidad de generar contextos educativos bilingües para los niños sordos. Pero también hay que tener en cuenta las ventajas de todo bilingüismo, que son positivas en el desarrollo integral del alumnado oyente.

Nuestro centro recoge en su proyecto educativo la particularidad de ser un centro bilingüe en el que todo nuestro alumnado, sea sordo u oyente, aprende la lengua castellana y la lengua de signos española. En este sentido, ambas lenguas son vehiculares para la comunicación y el 
aprendizaje y están presentes en todo momento en el centro. Este proyecto se lleva a cabo gracias a la organización y al compromiso de toda la comunidad educativa con las dos lenguas.

Nuestro modelo de educación compartida parte de una ratio que también es singular. Contamos con una media de 5 alumnos sordos de un total de 20 alumnos por aula. La agrupación de un número mayor de niños sordos en el aula facilita la interacción entre iguales, y permite que el niño sordo bilingüe no se encuentre solo en una clase de oyentes, pudiendo tener interlocutores signantes de su misma edad. En este contexto, llevar audífono, tener un implante coclear, o expresarse en lengua de signos, se percibe por el colectivo de manera normal. También favorece una mayor seguridad y desarrollo de la identidad personal y aumenta la imagen positiva de sí mismo. La agrupación permite, además, una mejor utilización de los recursos humanos y técnicos. El modelo implica que pueden organizarse actividades de aprendizaje en pequeños grupos para estimular la comunicación y cooperación entre todos los alumnos.

\section{Dos tutores por aula}

Para poder abordar toda esta diversidad nuestro centro apostó por una organización docente diferente a la que habitualmente encontramos en otros colegios. En el modelo que pusimos en marcha, inspirado en otros ya existentes (Rodríguez, 2005; Domínguez y Alonso, 2004) incorporamos al tradicional maestro de apoyo o PT dentro del aula, de tal forma, que en todos los cursos contamos con dos tutores. La finalidad de estos dos tutores cubre la doble función de poder atender con calidad a toda la diversidad en sentido amplio que aparece en nuestras aulas y además poder ser fieles al modelo bilingüe que proponemos en nuestro proyecto educativo. Así, contamos con una tutora referente en lengua castellana ("que habla") y otra tutora referente en lengua de signos española (LSE) ("que signa"). Las dos tienen el mismo peso y comparten la misma responsabilidad en todo el proceso de enseñanza-aprendizaje del

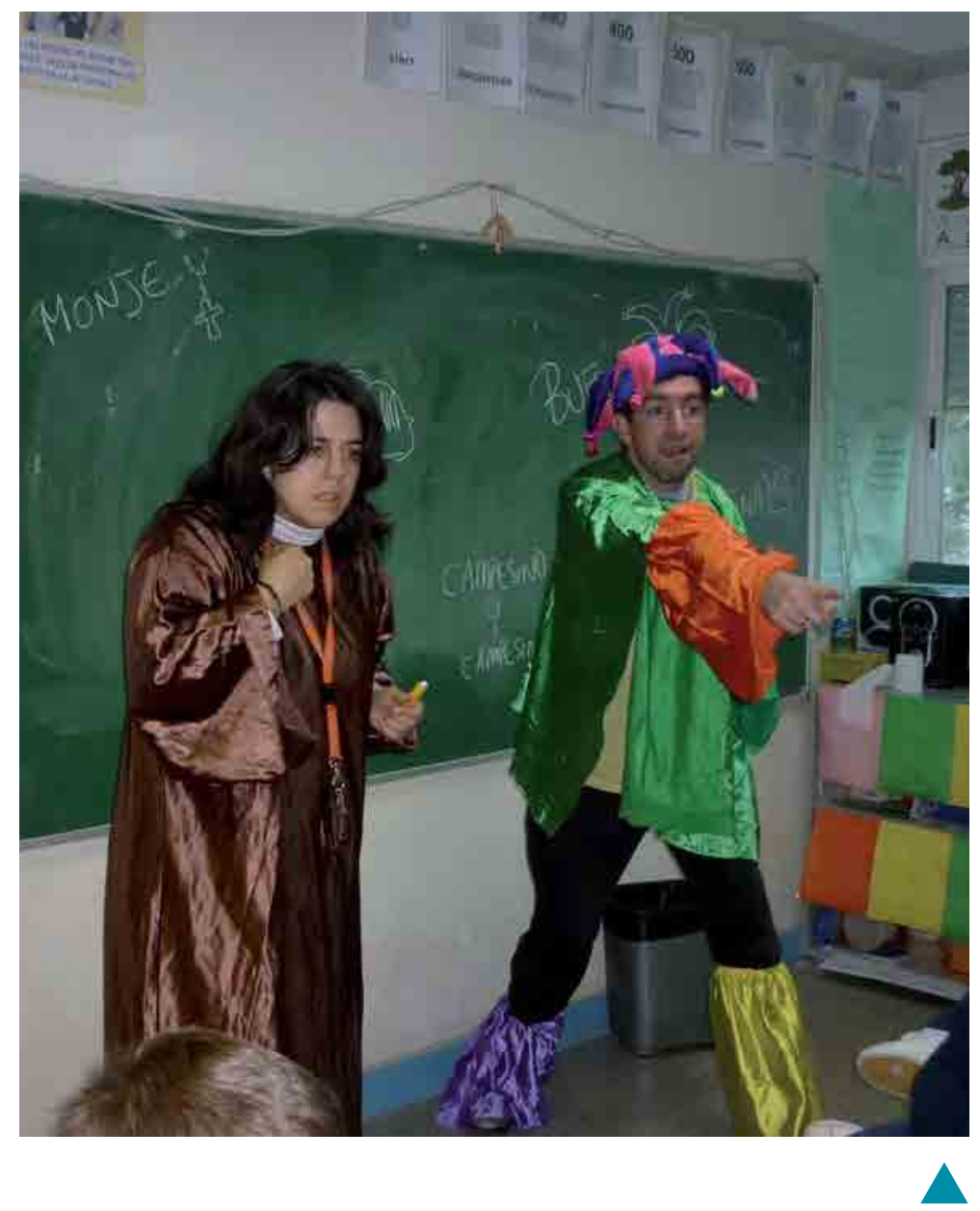

alumnado, sordo y oyente. Su presencia Dos lenguas en el aula. simultánea en el aula, apoyada sobre un importante trabajo colaborativo previo en la programación didáctica, y sobre un estatus profesional igual (no es que uno sea el tutor y el otro el "apoyo", sino que se trata de dos verdaderas tutoras), hace posible que todos estos alumnos estén juntos, reconociendo su diversidad y aprendiendo en función de sus capacidades y necesidades.

En este camino los tutores no están solos, contamos con otros especialistas que son fundamentales para conseguir una adecuada respuesta educativa ajustada a todos. Por una parte, contamos con el especialista en LSE que es un profesional sordo signante. Su función es favorecer la adquisición y competencia en LSE para todo el alumnado y ser modelo de identificación para los alumnos sordos. Desarrolla y participa en distintas actividades a lo largo de la jornada escolar.

\section{Agrupamientos flexibles}

Este modelo facilita que puedan organizarse actividades de aprendizaje con diferentes formatos de agrupación. Pode- 


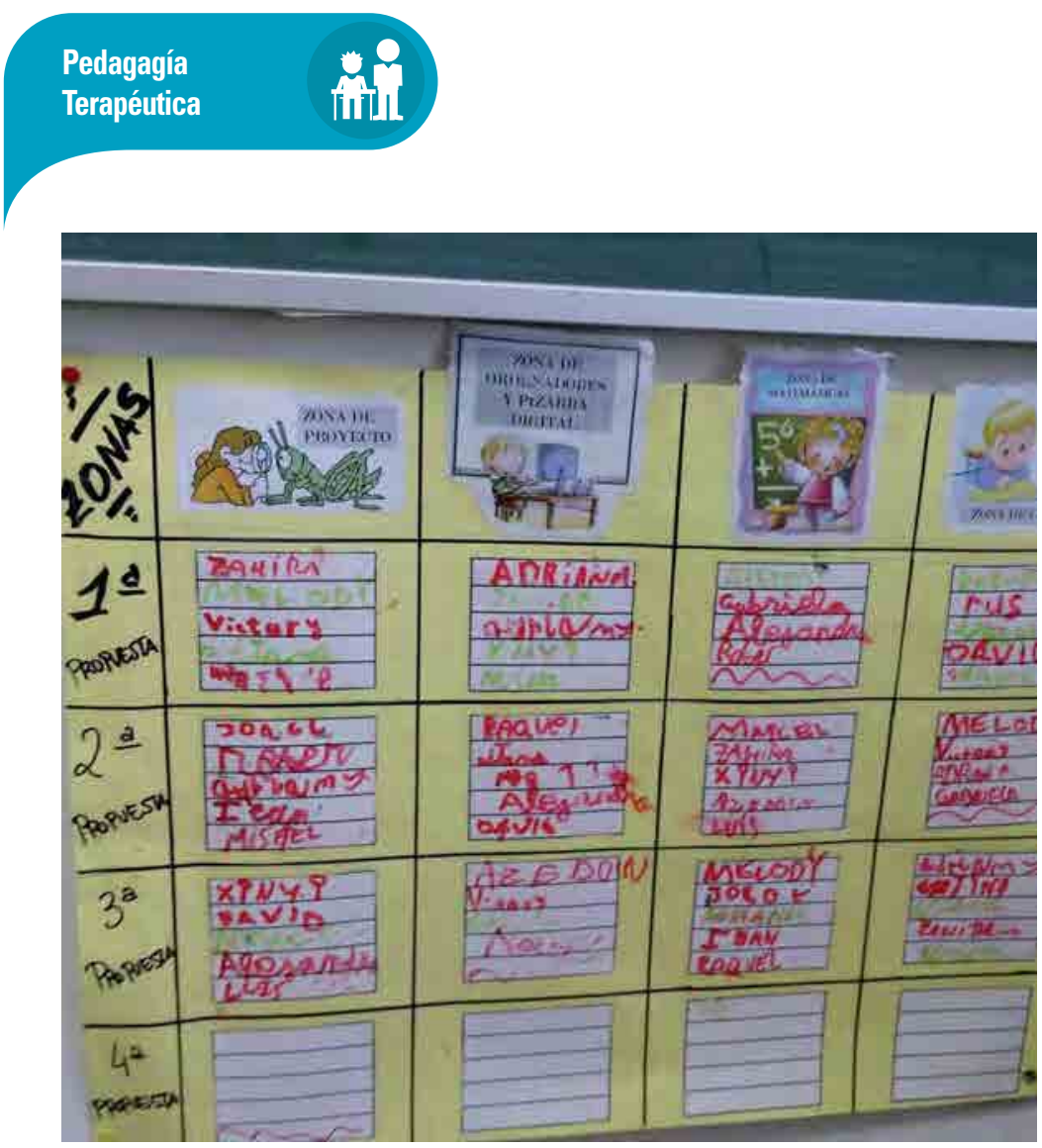

de dotar al alumnado que lo necesite, en cada momento, de las estrategias y procesos necesarios para poder incorporarse dentro del aula con el resto de sus compañeros.

Todos estos agrupamientos los realizan las tutoras, ya que son las que mejor conocen las necesidades del alumnado y, por lo tanto, pueden ajustar mejor la programación de los apoyos.

\section{La apuesta por metodologías flexibles}

Algunas de las claves de nuestra metodología son conseguir la autonomía, sensación de competencia, motivación hacia el aprendizaje, conexión entre el aprendizaje en la escuela y la realidad, oportunidades de colaboración para construir el conocimiento, aumentar las habilidades sociales. Todas ellas vinculadas a la necesidad de conseguir estudiantes competentes en las dos lenguas de nuestro centro.

En este sentido, las iniciativas metodológicas que hemos ido poniendo en marcha están encaminadas a la consecución de estos objetivos. Pasamos a comentar, a continuación, los dos pilares fundamentales de nuestra metodología que hacen posible todo lo anterior:

mos hacer actividades de gran grupo en las que todos los alumnos pueden participar al mismo tiempo porque reciben el mensaje en las dos lenguas que coexisten en el aula. Otras veces, preferiremos realizar agrupamientos de 4 a 6 alumnos que nos permiten estimular la comunicación y cooperación entre todos los alumnos y poder trabajar de manera más cercana con todos ellos. Y, además, a veces decidimos trabajar con un alumno concreto o una pareja para profundizar o explicar de forma más ajustada alguna cuestión.

La mayor parte del tiempo, estos diferentes tipos de agrupamientos ocurren dentro del aula, pero, en otras ocasiones decidimos que es necesario que algún alumno concreto o pequeños grupo salga del aula para poder trabajar procesos específicos que, de otra forma, sería complicado trabajar dentro de aula. El objetivo de estas agrupaciones es tratar

入 Proyectos de investigación. Partimos de la base de que el conocimiento se construye como un proceso de interacción entre la información procedente del medio y la que el estudiante posee, a partir de la que se inician nuevos conocimientos. Con esta metodología los alumnos se convierten en protagonistas de sus propios aprendizajes y éstos pueden integrarse en su estructura cognitiva porque son motivadores, tienen un contenido significativo y parten de sus conocimientos previos. En esta metodología, los recursos didácticos cumplen la finalidad de estimular y desarrollar las capacidades físicas, afectivas, intelectuales y sociales. Las nuevas tecnologías son para los niños tan novedosas como cualquier otro recurso de aprendizaje, por lo que se convier- 

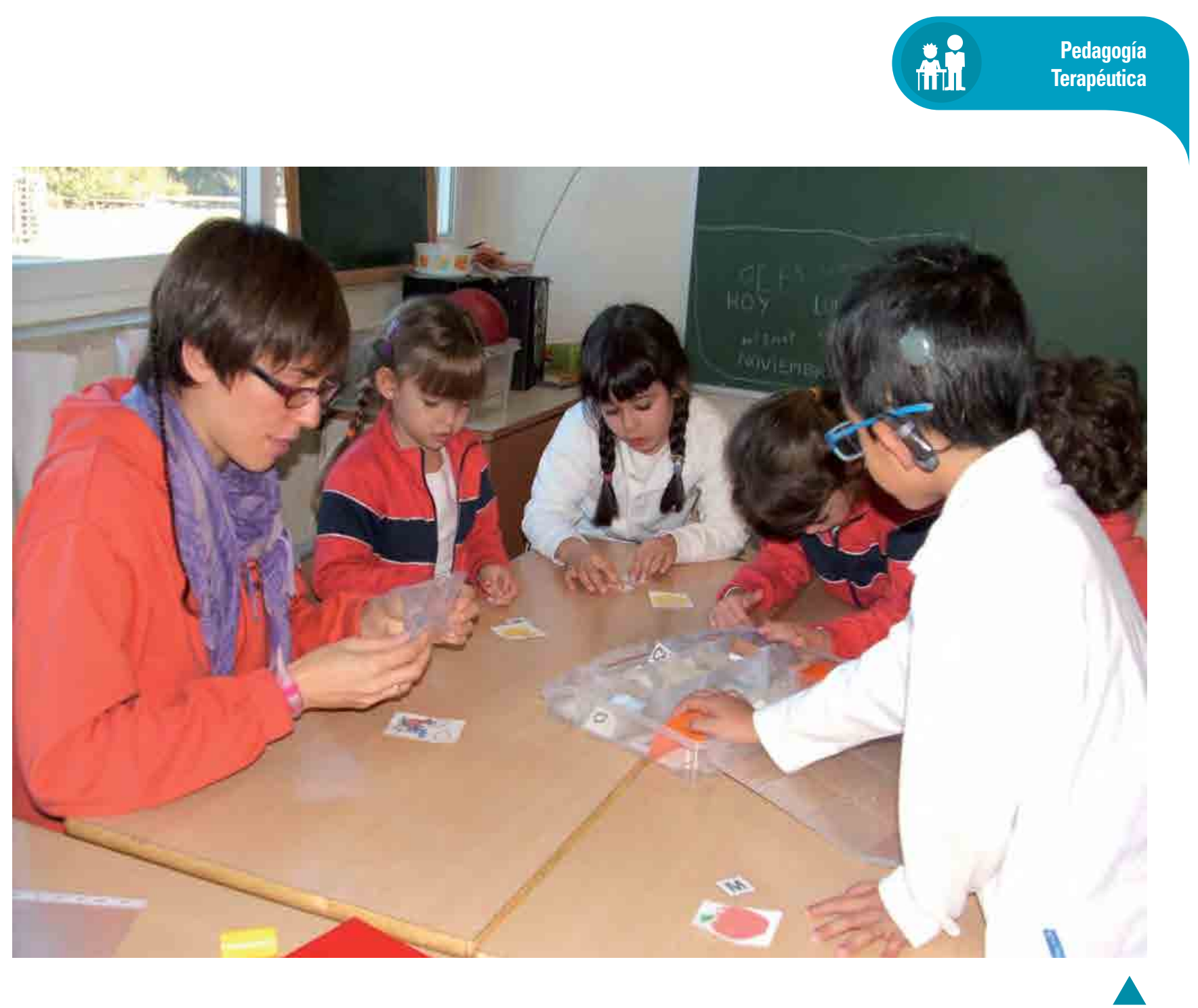

ten en una herramienta más para favorecer el desarrollo.

入 Zonas o rincones: en ambas etapas organizamos en aula por zonas o rincones. Es una propuesta metodológica que hace posible la participación activa del alumnado en la construcción de sus conocimientos. Son los estudiantes los que deciden a dónde irán en primer lugar, qué materia o actividad desarrollarán en cada momento. El aula se distribuye en varias zonas (Primaria) o rincones (Infantil) y los alumnos deciden libremente qué quieren aprender, experimentar, manipular.

\section{Las zonas como respuesta educativa en contextos de diversidad}

Esta metodología supone una doble ventaja. Por una parte, conseguimos que los alumnos que tiene más dificultades tengan mayores oportunidades para comunicarse y aportar sus soluciones en los problemas de aprendizaje. En el "gran grupo" a todos nos cuesta más levantar la mano y exponernos delante de nuestros compañeros y maestros, sin embargo, en "pequeño grupo" nos sentimos con más confianza para poder aportar nuestras ideas, pedir ayuda y colaborar en la elaboración de un trabajo.

A principio de curso, cada aula define sus cuatro zonas de trabajo, a las que les damos un nombre que nos guste y nos motive a todos y que identifique claramente lo que vamos a aprender en esa zona. En estas cuatro agrupaciones siempre están presentes lengua, matemáticas y proyecto. La cuarta zona, dependiendo de las edades y necesidades de cada grupo (autonomía, ritmo de aprendizaje), puede estar dedicada al área de educación artística (especialmente, expresión plástica) o al uso de las nuevas tecnologías (pizarra digital, tablet y PC).

Para poder llevar a cabo las zonas establecemos cuatro horas a la semana. En cada una de estas sesiones, se proponen cuatro actividades diferentes correspondientes con las cuatro zonas. Los estudiantes eligen, al inicio de la sesión, la zona en la que quieren trabajar ese día. La única condición es que deben pasar por las cuatro zonas a lo largo de la
Trabajamos en grupos pequeños para poder ajustar las ayudas. 

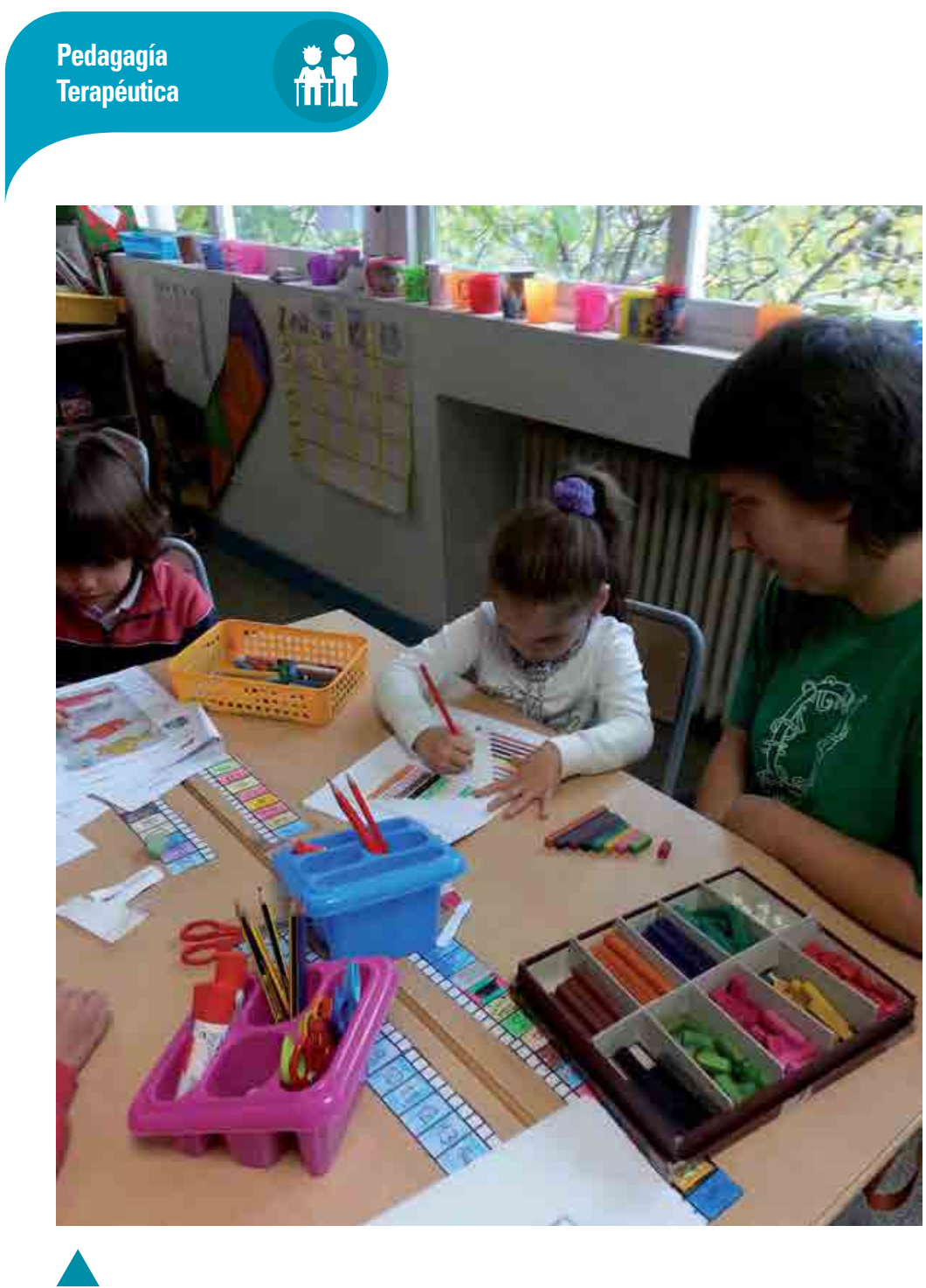

Ajustamos la ayuda a las características de nuestro alumnado. semana. De esta forma, el alumno puede elegir cada día aquello en lo que más le apetece trabajar. Todos tenemos días en los que nos sentimos con más ánimo para trabajar en un tipo de actividades que en otros. Se trata de respetar esos cambios en los estados de ánimo y motivacionales de nuestros estudiantes.

En cada zona hacemos una propuesta de trabajo con diferentes niveles o grados de dificultad que contempla la posibilidad de que cada alumno se enfrente a ella dentro de su zona de desarrollo próximo (ZDP). De esta forma, permitimos que el alumno, poco a poco, se vaya autorregulando hasta elegir la opción que mejor responde a sus necesidades e intereses en ese momento.

\section{El rol del maestro en las zonas}

Esta forma de trabajo, no sólo implica un cambio en la organización del aula, sino que, además, implica una transformación en nuestra forma de ver la educación y en nuestra actitud hacia el alumnado.

Los tutores en base a lo programado se reparten las zonas, es decir, donde se van a sentar para intentar dar respuesta a las

\author{
La flexibilidad es una de las \\ características fundamentales \\ de nuestros maestros y \\ maestras
}

necesidades de los alumnos. Dada esta organización, tenemos profesores que ayudan a los alumnos y alumnos que ayudan a otros alumnos. Con este formato, los apoyos no sólo se dan fuera del aula, sino que se ocurren también dentro lo que permite dar una mejor y más ajustada respuesta.

La flexibilidad es una de las características fundamentales de nuestros maestros y maestras; es decir, se acomodan con facilidad a las distintas situaciones que se dan en el aula y muestran una actitud de escucha activa hacia todo lo que propongan los alumnos, ya que por y para ellos estamos en las aulas. Por tanto, debemos tener la capacidad de alternar entre distintos criterios necesarios para atender las demandas que cada día van a ser cambiantes de nuestras situaciones de enseñanza y aprendizaje.

De esta manera, los maestros pueden adaptarse al máximo al alumnado, a sus diferentes ritmos de aprendizaje y características personales, convirtiéndose así, en apoyos eficaces para todos los alumnos que lo necesiten. Además, los maestros son los encargados de coordinar, regular y controlar los tiempos en las sesiones de zonas.

Esta organización nos permite, como hemos mencionado anteriormente, un trabajo más especializado con aquellos alumnos que requieren de un mayor apoyo, lo que implica una actitud abierta ante las necesidades y la formación en las mismas, constante y dinámica, buscando siempre las propuestas que mejoren el ritmo de aprendizaje de los alumnos.

Los maestros se convierten, por tanto en facilitadores, dinamizadores y su- 

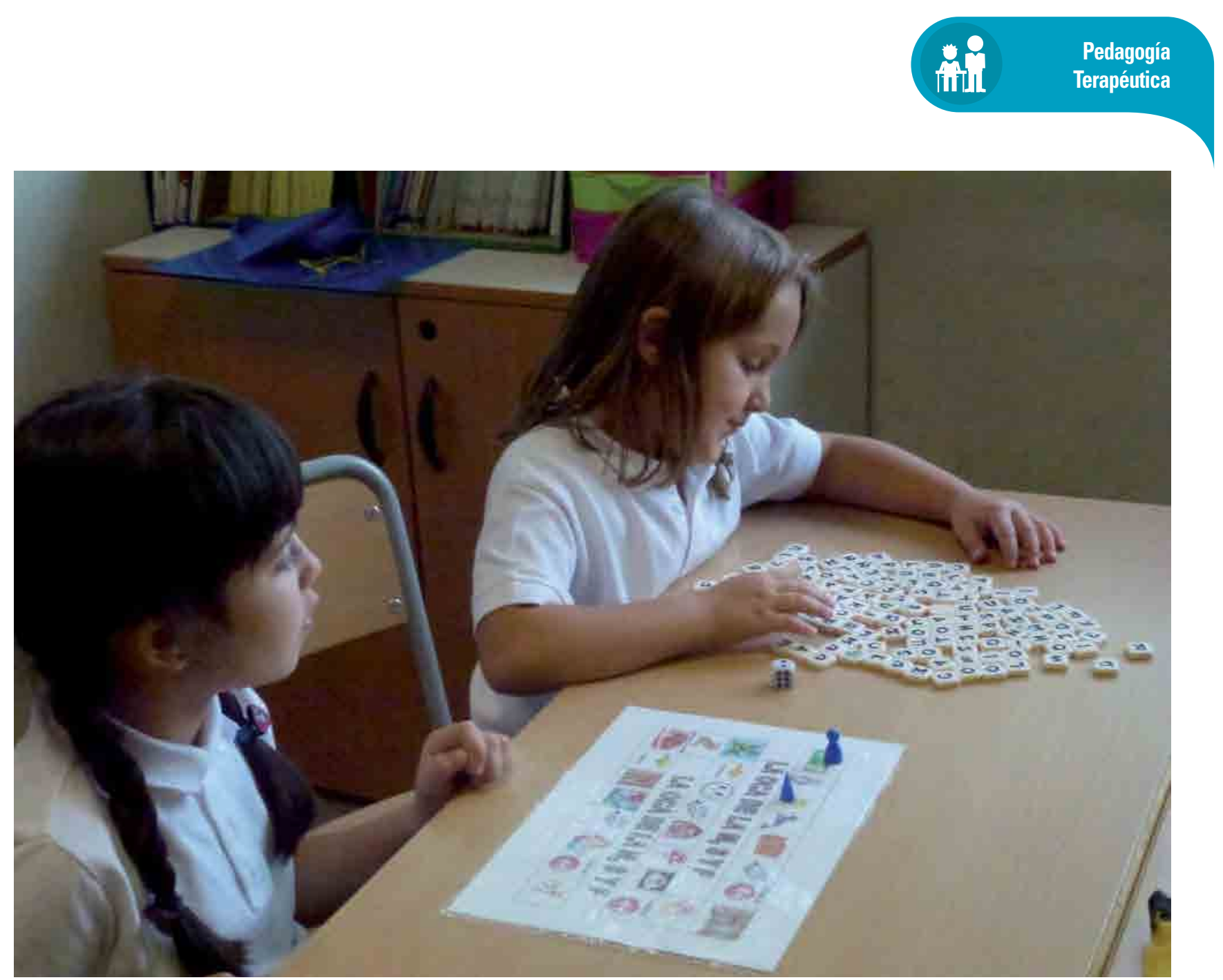

pervisores de las tareas, proporcionando situaciones de aprendizaje favorables, adecuadas, funcionales y significativas para promover el aprendizaje por descubrimiento y autónomo. Para todo ello es primordial el trabajo en equipo, y una autoevaluación constante de nuestra tarea como educadores. El maestro es una clave esencial en este trabajo, saben buscar y cuidar los momentos de reflexión y el análisis de sus prácticas para una mejora constante.

\section{Materiales y recursos didácticos en el trabajo por zonas}

A la hora de diseñar nuevas propuestas para las zonas tenemos en cuenta algunos aspectos que comentamos a continuación:

ע Todos los materiales van siempre acompañados de unas orientaciones (en forma de instrucciones) suficientes para que los niños de forma autónoma puedan saber lo que tienen que hacer en cada momento.

$\checkmark$ Materiales diversos que sirvan para que cada niño pueda elegir la estra- tegia más adecuada a sus características.

ע Propuestas que permitan la conexión entre distintas materias con el proyecto como hilo conductor, de tal forma que se fomente un aprendizaje significativo y global. Al trabajar por proyectos de investigación todas las zonas se intentan relacionar con el proyecto que se está trabajando.

En este sentido, los materiales serán diversos y variados. Proponemos algunos ejemplos:

ע Materiales de consulta (libros de texto y cuadernos de todo tipo editados con finalidades académicas y curriculares). Uno de los materiales de consulta estrella en el trabajo por proyectos llamado Libro importante. El Libro importante es nuestro cuaderno de bitácora de los proyectos de investigación, una especie de guía de aprendizaje, la herramienta que nos sirve para ir organizando los conocimientos que vamos construyendo entre maestros y estudiantes. En él vamos incorporando la 


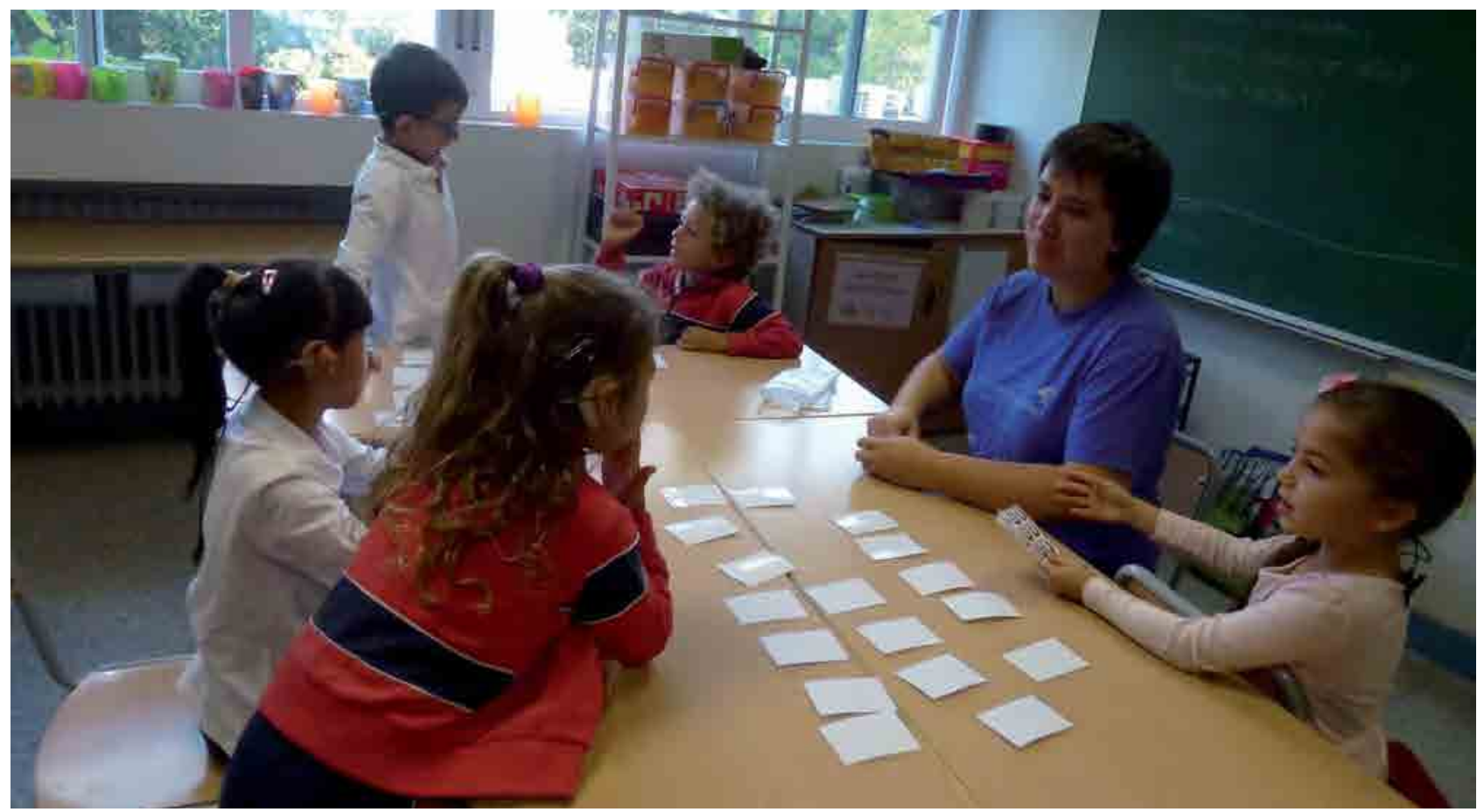

Trabajar en pequeño grupo nos permite dar una respuesta más especializada teoría que estudiamos en cada una de las materias (lengua, matemáticas, ciencias sociales...) adaptada al momento en el que se encuentra cada alumno. Algunos alumnos necesitan más apoyo visual, otros frases más sencilla, etcétera.

y Biblioteca de aula, del centro y libros que aportan maestros y alumnos: libros, cuentos, cómics, libros de consulta, prensa.

$\checkmark$ Tablet, PC, pizarra digital, ordenadores portátiles. Las nuevas tecnologías les Ilaman la atención y les

\section{(D.) DARA SABER MÁS}

Domínguez, A. y Alonso P. (2004). La educación de los alumnos sordos hoy. Perspectivas y respuestas educativas. Málaga: Aljibe.

Acosta, V. (2006) (dir.). La sordera desde la diversidad cultural y lingüística. Construyendo centros inclusivos en la educación del alumnado con sordera. Barcelona: Masson.

Enlaces web:

Blog del aula de $2^{\circ}$ de EP: http://generacion2014investigando. blogspot.com.es/

Blog del aula de $3^{\circ}$ de EP: http://generacion2013investigando. blogspot.com.es/

Blog del aula de la etapa de Infantil: http:// elpasillodeabajo.blogspot.com.es/ atraen mucho, además de suponer un gran apoyo visual.

\ Materiales didácticos específicos para las distintas áreas: tales como el multibase (material muy concreto para trabajar las matemáticas que se componen de diferentes piezas de unidades, decenas y centenas), Numicon, (mediante un enfoque multisensorial ayuda a entender a los alumnos la idea de número y de relaciones numéricas), varillas, dominós, bingos, Memory.

\ Materiales elaborados por los propios maestros para cubrir los objetivos propuestos en cada zona. Al no tener libros de texto los maestros elaboran tanto los materiales de zona como las teorías. Esto permite que el alumno vea el proceso de enseñanza aprendizaje como algo cercano a sus intereses y además nos permite elaborar materiales ajustados a cada uno •

\section{HEMOS HABLADO DE}

\section{Pedagogía terapéutica; discapacidad auditiva; PT; mirada; problemas de aprendizaje.}

Este artículo fue solicitado por PADRES y MAESTROS en septiembre de 2015, revisado y aceptado en enero de 2016. 


\section{Eurobecas FP Montemadrid}

Un camino seguro

para las prácticas en el extranjero

Desde 1998, 3000 estudiantes de FP han realizado prácticas en el extranjero gracias a Fundación Montemadrid.

Además, este año se incorpora el proyecto De Madrid a Irlanda Erasmus+ FP, gestionado por Fundación Montemadrid y financiado por fondos europeos. Erasmus+ 